\title{
HIGHER RANK NUMERICAL HULLS OF MATRICES AND MATRIX POLYNOMIALS
}

\author{
GHolamreza Aghamollaei AND SharifeH REZAgholi
}

\begin{abstract}
In this paper, some properties of the higher rank numerical hulls, as a generalization of higher rank numerical ranges and polynomial numerical hulls, of matrices are investigated. In particular, the higher rank numerical hulls of Pauli matrices are characterized. Moreover, the notion of higher rank numerical hulls of matrix polynomials is introduced, and some algebraic properties of this notion are investigated. The higher rank numerical hulls of the basic $A$-factor block circulant matrix, which is the block companion matrix of the matrix polynomial $Q(\lambda)=$ $\lambda^{s} I_{n}-A$, are also studied.
\end{abstract}

Mathematics subject classification (2010): 15A60, 15A18, 15A69, 47A56, 81P68.

Keywords and phrases: Higher rank numerical hull, rank- $k$ numerical range, joint higher rank numerical range, polynomial numerical hull, Pauli matrices, matrix polynomials.

\section{REFERENCES}

[1] Gh. Aghamollaei And A. SAlemi, Polynomial numerical hulls of matrix polynomials, II, Linear Multilinear Algebra, 59 (2011), 291-302.

[2] A. ARetaki AND J. Maroulas, The higher rank numerical range of matrix polynomials, Preprint available at http://arxiv.org/abs/1104.1328.

[3] M. BAyat, H. Teimoori AND B. Mehri, A generalization of rotations and hiperbolic matrices and its applications, Electr. J. Linear Algebra, 16 (2007), 125-134.

[4] L. D. Berkovitz, Convexity and Optimization in $\mathbb{R}^{n}$, John Wiley \& Sons, Inc. 2002.

[5] M. D. Choi, M. Giesinger, J. A. Holbrook And D. W. Kribs, Geometry of higher rank numerical ranges, Linear Multilinear Algebra, 56 (2008), 53-64.

[6] M. D. ChOI, D. W. KRIBS AND K. ZYCZKOWS KI, Higher rank numerical ranges and compression problems, Linear Algebra Appl. 418 (2006), 828-839.

[7] Ch. Davis, C. K. Li ANd A. SAlemi, Polynomial numerical hulls of matrices, Linear Algebra Appl. 428 (2008), 137-153.

[8] Ch. Davis AND A. SAlemi, On polynomial numerical hulls of normal matrices, Linear Algebra Appl. 383 (2004), 151-161.

[9] M. Eiermann, Field of values and iterative methods, Linear Algebra Appl. 180 (1993), 167-197.

[10] V. Faber, A. Greenbaum And D. E. Marshall, The polynomial numerical hulls of Jordan blocks and related matrices, Linear Algebra Appl. 374 (2003), 231-246.

[11] I. Gohberg, P. Lancaster and L. Rodman, Matrix Polynomials, Academic Press, New York, 1982.

[12] A. Greenbaum, Generalizations of field of values useful in the study of polynomial functions of a matrix, Linear Algebra Appl. 347 (2003), 233-249.

[13] A. Greenbaum, Upper and lower bounds on norms of functions of matrices, Linear Algebra Appl. 430 (2009), 52-65.

[14] K. E. Gustafson And D. K. M. RaO, Numerical Range: The Field of Values of Linear Operators and Matrices, Springer-Verlage, New York, 1997.

[15] R. Horn And C. Johnson, Topics in Matrix Analysis, Cambridge University Press, New York, 1991. 
[16] E. Knill and R. Laflamme, Theory of quantum error-correcting codes, Phys. Rev. A. 55 (1997), 900-911.

[17] E. KNill, R. Laflamme AND L. Viola, Theory of quantum error correction for general noise, Phys. Rev. Lett. 84 (2000), 2525-2528.

[18] D. W. Kribs, R. Laflamme, D. Poulin And M. Lesosky, Operator quantum error correction, Quant. Inf. Comput. 6 (2006), 383-399.

[19] C. K. Li AND Y. T. Poon, Generalized numerical ranges and quantum error correction, J. Operator Theory, 66 (2011), 335-351.

[20] C. K. LI, Y. T. Poon AND N. S. SzE, Condition for the higher rank numerical range to be non-empty, Linear Multilinear Algebra, 57 (2009), 365-368.

[21] C. K. LI, Y. T. Poon AND N. S. SZE, Higher rank numerical ranges and low rank perturbations of quantum channels, J. Math. Anal. Appl. 348 (2008), 843-855.

[22] C. K. Li And L. Rodman, Numerical range of matrix polynomials, SIAM J. Matrix Anal. Appl. 15 (1994), 1256-1265.

[23] O. Nevanlinna, Convergence of Iterations for Linear Equations, Birkhäuser, Basel, 1993.

[24] O. Nevanlinna, Hessenberge matrices in Krylove subspace and computation of the spectrum, $\mathrm{Nu}-$ mer. Func. Anal. Optim. 16 (1995), 443-473.

[25] M. A. Nielsen And I. L. ChuAng, Quantum Computation and Quantum Information, Cambridge University Press, 2010.

[26] A. SALEMI, Higher rank numerical hulls of matrices, Operators and Matrices, 6 (2012), 79-84.

[27] A. Salemi And Gh. Aghamollaei, Polynomial numerical hulls of matrix polynomials, Linear Multilinear Algebra, 55 (2007), 219-228. 